\title{
Preserving last resort antibiotics: A meropenem reduction strategy
}

\author{
Geraldine M. Conlon-Bingham $\mathrm{PhD}^{1}$ (1), Sara A. Hedderwick FRCP2 ${ }^{2}$ Cara M. McKeating FRCPath ${ }^{2}$, \\ Peter M. McKee MSc ${ }^{1}$, Jessie C. McNally MPharm¹, Lisa M. Lennon MPharm¹, Orla McGivern MSc ${ }^{1}$, \\ Kevin Lewis MBChB², Dominic McKenna MBChB ${ }^{3}$, Elizabeth A. Lattyak PharmD ${ }^{4}$, William J. Lattyak BSc ${ }^{4}$ and
} Mamoon A. Aldeyab $\mathrm{PhD}^{5}$

${ }^{1}$ Pharmacy Department, Southern Health and Social Care Trust, Craigavon, Northern Ireland, United Kingdom, ${ }^{2}$ Microbiology Department, Southern Health and Social Care Trust, Craigavon, Northern Ireland, United Kingdom, ${ }^{3}$ ENT Department, Craigavon Area Hospital, Craigavon, Northern Ireland, United Kingdom, ${ }^{4}$ Scientific Computing Associates, River Forest, Illinois, United States and ${ }^{5}$ Department of Pharmacy, School of Applied Sciences, University of Huddersfield, Huddersfield, United Kingdom

To the Editor-Carbapenems are a group of broad-spectrum antibiotics that have activity against a range of resistant bacteria. In line with the UK government targets, the Northern Ireland Executive has mandated Health Trusts to reduce total antibiotic prescribing by $15 \%$ by $2023 .{ }^{1}$ Restrictive interventions are one tool to help achieve these targets; their effectiveness in reducing antibiotic prescribing when the need is urgent has been demonstrated. However, both restrictive and persuasive interventions are equally effective in the longer term. ${ }^{2}$ More recently, persuasive interventions, in particular those involving "handshake stewardship" have been shown to be more effective than restrictive interventions in reducing not only antibiotic consumption but also in reducing the incidence of bacteremia caused by carbapenem-resistant gram negative bacilli. ${ }^{3}$

This study was undertaken in 2 district general hospitals in the Southern Health and Social Care Trust (SHSCT), Daisy Hill Hospital (DHH) and Craigavon Area Hospital (CAH). The quasi-experimental interrupted time series (ITS) design involved 3 phases: First, an audit of how meropenem was prescribed in the SHSCT (September-December 2018). This phase involved a review of the case notes for all patients who received meropenem to identify duration of therapy, indication of meropenem therapy, demographic data, and relevant laboratory tests. The second phase involved the implementation of weekly meropenem ward rounds led by the antimicrobial stewardship team (AST; June 2019February 2020). During this period, e-mail alerts detailing patients for whom meropenem was ordered were sent to the antimicrobial pharmacists. An initial review of these patients was undertaken, and a timeline of the infection course was prepared. The meropenem ward round was then undertaken by the AST; it involved an assessment of the patient and discussion with the medical team to determine the best course of action for the management of the infection. The third phase of this project resulted from the suspension of the meropenem ward rounds due to the coronavirus disease 2019 (COVID-19) pandemic (March-December 2020). The effect of the introduction and subsequent suspension of the ward rounds on meropenem consumption in both hospitals was evaluated using ITS analysis. ${ }^{4}$ The study was registered as a quality improvement project in the SHSCT.

Author for correspondence: Geraldine M. Conlon-Bingham, E-mail: geraldine. conlonbing@southerntrust.hscni.net

Cite this article: Conlon-Bingham GM, et al. (2022). Preserving last resort antibiotics: A meropenem reduction strategy. Infection Control \& Hospital Epidemiology, 43: 1516-1517, https://doi.org/10.1017/ice.2021.276
The results of the initial meropenem audit demonstrated that $69 \%$ of prescriptions were recommended following a telephone consultation with the microbiology department. For $92 \%$ of patients, antibiotic therapy was continued following the 72-hour antibiotic review. The average duration of meropenem therapy was 11 days. Between June 19 and February 20, the AST-led meropenem rounds were implemented in $\mathrm{DHH}$ and CAH. Reviews of therapy were conducted for 75 patients. In $32 \%$ of patients, a recommendation was made to stop meropenem. In a further $25 \%$ of patients, a recommendation was made to switch to an alternative antibiotic. A date to stop meropenem therapy was recommended in $15 \%$ of patients and in $28 \%$ of patients a recommendation was made to continue treatment. The segmented regression analysis of meropenem consumption demonstrated that, for the intervention period (June 2019February 2020), a significant decrease in meropenem consumption was observed (change in level: coefficient $=-4.6809$; $P<.001)$. The suspension of the carbapenem ward rounds because of the COVID-19 pandemic did not significantly impact the level or trend in carbapenem consumption (Fig. 1).

ID physician reviews of carbapenem prescribing have been shown to lead to a reduction in the use of these antibiotics. In addition, reductions in duration of therapy, cost, inpatient days, and 30-day mortality have been demonstrated. ${ }^{5-8}$ Decreases in carbapenem resistance in $P$. aeruginosa have also been observed because of an infectious diseases consultation intervention. ${ }^{9}$ Following the implementation of the meropenem ward rounds, recommendations were made to stop this antibiotic in $32 \%$ of patients, despite these uses being initiated on the advice of the microbiologist. This finding highlights the value of an antimicrobial pharmacist undertaking initial reviews of these patients followed by an independent assessment by the consultant microbiologist or ID physician. As a result of the COVID-19 outbreak, the meropenem ward rounds were suspended, highlighting the impact of the pandemic on antibiotic stewardship activities. Nevertheless, no significant change in the trend of meropenem consumption was observed. During the meropenem ward rounds, case discussions were undertaken between the AST and the clinical team (junior medical staff, ward pharmacists, middle grade medical staff and consultants). These exchanges provided the opportunity to educate clinical staff on the management of infection and may have contributed to the observed sustained reduction in meropenem consumption. This communication may also have contributed to the lack of increase in consumption of this antibiotic, once the ward 


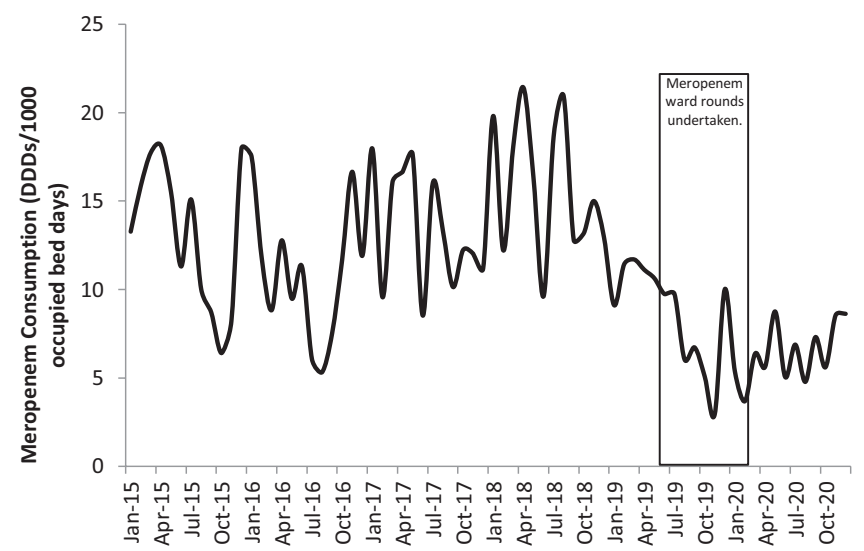

Fig. 1. Southern Health and Social Care Trust (SHSCT) meropenem consumption between January 2015 and December 2020; vertical lines represent the intervention period (June 2019-February 2020).

rounds were suspended. Previous studies have demonstrated the long-term benefits of the "handshake stewardship" approach in pediatric healthcare settings. ${ }^{9,10}$ MacBrayne et $\mathrm{al}^{9}$ observed decreases on total antibiotic use for up to 5 years after implementing an intervention consisting of ID physician or antimicrobial pharmacist review of patients regarding antibiotic consumption. ${ }^{9}$ The in-person approach used in the present study allowed a full patient assessment to be performed followed by face-to-face conversations with the clinical team in a nonconfrontational manner.

This study demonstrates the importance of an in-person review by the AST team, which has enabled rationalizing of antibiotic therapy, resulting in significant reductions in meropenem prescribing. The AST-led meropenem reviews also produced added benefits through the education of clinical teams following faceto-face case discussions, which have the potential to have long-lasting effects beyond the duration of the intervention.

Acknowledgments. This project was considered a service evaluation, and ethical approval was not required. This study was registered with the Health Trust audit department.
Financial support. This study was carried out as part of our routine work.

Conflicts of interest. All authors report no conflicts of interest relevant to this article.

\section{References}

1. NI Department of Health, Department of Agriculture, Environment and Rural Affairs, Foods Standards Agency. Changing the culture 2019-2024: one health. Tackling antimicrobial resistance in Northern Ireland: a fiveyear action plan. Northern Ireland Department of Health website. https://www.health-ni.gov.uk/sites/default/files/publications/health/onehealth-antimicrobial.pdf. Published 2019. Accessed April 9, 2020.

2. Davey P, Brown E, Charani E, et al. Interventions to improve antibiotic prescribing practices for hospital inpatients. Cochrane Database Syst Rev 2013;(4):CD003543.

3. Moghnieh R, Awad L, Abdallah D, et al. Effect of a "handshake" stewardship program versus a formulary restriction policy on high-end antibiotic use, expenditure, antibiotic resistance, and patient outcome. J Chemother 2020; 32:368-384.

4. Jirjees FJ, Al-Obaidi HJ, Sartaj M, et al. Antibiotic use and resistance in hospitals: time-series analysis strategy for determining and prioritising interventions. Hosp Pharm Eur 2020;95:13-19.

5. Garcia-Rodriguez JF, Bardan-Garcia B, Pena-Rodriguez MF, Alveraz-Diaz H, Marino Callejo A. Meropenem antimicrobial stewardship program: clinical, economic, and antibiotic resistance impact. Eur J Clin Microbiol Infect Dis 2019;38:161-170.

6. Seah VXF, Ong RYL, Lim ASY, Chong CY, Tan NWH, Thoon KC. Impact of a carbapenem antimicrobial stewardship program on patient outcomes. Antimicrob Agents Chemother 2017;61:e00736-17.

7. Suzuki A, Maeda M, Yokoe T, Hashiguchi M, Togashi M, Ishino K. Impact of the multidisciplinary antimicrobial stewardship team intervention focusing on carbapenem de-escalation: a single-centre and interrupted time series analysis. Int J Clin Pract 2020;75:e13693.

8. Horikoshi Y, Suwa J, Higuchi H, et al. Sustained pediatric antimicrobial stewardship program with consultation to infectious diseases reduced carbapenem resistance and infection-related mortality. Int $J$ Infect Dis 2017;64:69-73.

9. MacBrayne CE, Williams MC, Levek C, et al. Sustainability of handshake stewardship: extending a hand is effective years later. Clin Infect Dis 2020; 70:2325-2332.

10. Hurst A, Child J, Parker S. Intervention and acceptance rates support handshake stewardship. J Pediatric Infect Dis Soc 2019;8:162-165.

\title{
Strategy to limit multidrug-resistant Acinetobacter baumannii transmission in a cohort coronavirus disease 2019 (COVID-19) critical care unit
}

\author{
Anucha Apisarnthanarak MD ${ }^{1}$ (1) and David J. Weber MD, MPH² \\ ${ }^{1}$ Division of Infectious Diseases, Thammasat University Hospital, Pathum Thani, Thailand and ${ }^{2}$ Gillings School of Global Public Health, Chapel Hill, \\ North Carolina, United States
}

\footnotetext{
Author for correspondence: Anucha Apisarnthanarak, Email: anapisarn@yahoo.com Cite this article: Apisarnthanarak A and Weber DJ. (2022). Strategy to limit multidrugresistant Acinetobacter baumannii transmission in a cohort coronavirus disease 2019 (COVID-19) critical care unit. Infection Control \& Hospital Epidemiology, 43: 1517-1518, https://doi.org/10.1017/ice.2021.289
}

To the Editor-Coinfection with multidrug-resistant organisms (MDROs) among coronavirus disease 2019 (COVID-19) patients is common in critical care patients with a prolonged length of stay in critical care units, likely due to the coadministration of high-dose steroids and the prolonged duration of mechanical ventilation. ${ }^{1}$ The control of MDROs among COVID-19 patients 